\title{
Benevolent Autocratic Opacity
}

\author{
Ying Xue \\ Rowan University
}

\begin{abstract}
The uncertainty of whether the benevolent sender is attentive to a matter reduces the decision-making receiver's welfare under democratic transparency. The sender restores efficiency by muzzling a dissident opinion that is as accurate as her own information. Since some uncertainty is inevitable, the sender replaces a more welfare-relevant uncertainty by a less welfare-relevant uncertainty. The paper has applications in politics, law, and economics with novel predictions and policy implications.
\end{abstract}

Keywords: disclosure, transparency, governance, autocracy, opacity, law, communication.

JEL Classification Code: D04, H11, H12, K29. 


\section{Introduction}

It seems puzzling that COVID-19 has taken a heavier toll on transparent democracies than opaque autocracies. Likewise, Winston Churchill skipped much bad news when he addressed the nation but managed to lead Britain to victory. Nevertheless, our society seems intolerant of surveillance and opacity. The Watergate scandal lead to the only resignation of a U.S. president. More recently, Edward Snowden's leaks put surveillance programs under debate. This paper shows that autocratic surveillance and opacity can maximize social welfare.

Our paper concerns how a benevolent sender can efficiently disclose information to the receiver to help him make an informed decision. The sender is busy and may not be attentive to a particular decision of the receiver. Even in the COVID-19 crisis, governments were at alarming levels of inaction and inattention according to the World Health Organization. Besides, not everything can make headlines. Governments and entities do not make frequent non-news announcements like "we have no information but we are attentive to the matter". However, when no announcement comes, the receiver cannot tell if the sender is inattentive to an issue or has no news.

When the sender may be inattentive, the receiver cannot rely on the sender to tell the states of nature that pool together in his information sets. The uncertainty of whether the sender is attentive remains for the receiver even if the sender is transparent about what she knows when she is attentive. This uncertainty impairs the efficiency of the receiver's decision because different decisions are optimal for different states in the same information set. Without the sender's intervention, the receiver makes wrong decisions in some states. The sender can restore efficiency by muzzling dissident opinions, which enables the receiver to resolve the more welfare-relevant uncertainty.

When the sender is attentive, she is the first to become informed of any news. For example, Grand Princess cruise passengers found out they had COVID-19 from Mike Pence's speech. The sender makes her official announcement if any, and decides whether to squash an equally accurate rumor before it spreads to the public. In equilibrium, the sender stops a rumor that disagrees with her official information even though both signals have the same information quality. Such autocratic opacity to eliminate dissident yet helpful information seems contradictory to the benevolent sender's objective to disclose more information to help the receiver, but it enables the receiver to distinguish key events. The side effect is introducing a new uncertainty of whether the informative rumor has been suppressed or it simply never existed. Fortunately, the new uncertainty has no bearing on decision-making or welfare. In essence, the sender substitutes a less relevant uncertainty for the key uncertainty that impacts the receiver's welfare.

Technically, the sender's intervention rearranges the receiver's information sets so that the same decision is unanimously optimal for all states in each information set. This enables the receiver to make the optimal decision in each state as if he received enough information to distinguish every state. In a way, the sender maximizes the receiver's welfare subject to an uncertainty constraint, and chooses the less relevant uncertainty. Overall, autocratic opacity attains efficiency because the loss of information does not lead to wrong decisions that reduce the receiver's welfare. 
The paper has applications in law, public policy, and political economy on the rights and boundaries of governments and the powers that be in caring for receivers facing a decision. Without authorities' warning, receivers cannot tell whether the authorities are inattentive or have no adverse information, such as whether the government was aware of risks in financial innovations in the great recession or actively monitoring the nation in a health crisis. We have witnessed receivers' decisions in pandemics to stay at home, get tested, use protective equipment, stock up on essentials and guns, take medicines under trial, and reopen businesses afterwards. The decision in general can be whether to take a certain option or behave in a certain way, both in crisis like terrorism, natural disasters, nuclear leaks and in normality such as choices in investments, foods, drinks, products, sports, entertainment, activities, and services. Regardless, authorities should be allowed surveillance to be the first to hear and stop dissident unofficial information from reaching the public to help them make efficient decisions.

Hirshleifer (1971) shows information has little social value unless it alters production. The sender in our paper substitutes more welfare-relevant information for less welfare-relevant information to enable the receiver to optimize his decision. Burguet and Vives (2000) and Amador and Weill (2012) show that public disclosure may discourage acquiring and learning information but neither effect exists in our paper. The team leader in a public-good game by Teoh (1997) withholds information to preempt coordination failures. Morris and Shin (2002) and Angeletos and Pavan (2007) show public disclosure may crowd out private information when there are motives to coordinate. Our paper is not about coordination, and our receiver has no private information.

Gonedes and Dopuch (1974) posits that the choice of accounting techniques reveals information. Gonedes (1980) finds that mandatory and voluntary disclosures can be substitutes. Dye (1985b) builds on both points against mandating disclosure to show that firms need to withhold proprietary information. We offer another reason against mandated transparency because our sender discloses useful information by withholding less useful information. Dye (1986) shows optimal withholding when managers have both proprietary and non-proprietary information. Dye (1985a) offers additional rationales for managers' reticence due to investors' incomplete knowledge of managers' information and the principal-agent problem between shareholders and managers.

Grossman (1981), Milgrom (1981), and Grossman and Hart (1980) show the unraveling result where firms disclose credibly and voluntarily to separate from worse types. Unraveling does not take place in our paper even though the sender's interest is perfectly aligned with the receiver's and more information helps the receiver. This is because when the sender can be inattentive, some uncertainty is inevitable, and the sender substitutes less welfare-relevant uncertainty for more welfare-relevant uncertainty to improve the receiver's welfare. A less biased sender adds less noise to the original state variable in the cheap talk game by Crawford and Sobel (1982). Some noise is inevitable in our paper, and the unbiased sender attains efficiency by swapping out the more welfare-relevant uncertainty.

The rest of the paper proceeds as follows. The next section sets up our model and derives the first-best. Section 3 shows inefficient democratic transparency and Section 4 shows efficient autocratic opacity. Section 5 presents the predictions, policy 
implications, and the robustness of our analysis. The final section concludes.

\section{The Model}

There are two parties, the benevolent sender $(S$, she) and the receiver $(R$, he). $R$ decides whether to take an option $\tilde{x}$, which is a priori equally likely to be good $(g)$ or bad (b) for him. $R$ gains 1 from a good $\tilde{x}$ but loses $c>0$ from a bad $\tilde{x}$. $R$ 's payoff from avoiding $\tilde{x}$ is $0 . R$ maximizes his expected payoff by either having $\tilde{x}$ or avoiding it. $S$ is busy but hopes to send $R$ information to help him maximize welfare. Independent of other randomness, $S$ is attentive $(a)$ to $R$ 's decision with probability $p \in(0,1)$ but is inattentive $(i)$ otherwise. $p$ may represent $S$ 's conscientiousness, bandwidth, responsiveness, or ability.

Two sources of information may aid $R$ in his choice. First, if $S$ is attentive, she issues an official warning $w$ that $\tilde{x}$ is bad whenever she detects one. $w$ appears according to

$$
\operatorname{Pr}(w \mid \tilde{x}=b)=q>\frac{1}{2}, \operatorname{Pr}(w \mid \tilde{x}=g)=1-q>0,
$$

where $q$ signifies information quality. Second, a rumor $r$ that $\tilde{x}$ is bad comes into existence according to

$$
\operatorname{Pr}(r \mid \tilde{x}=b)=q, \operatorname{Pr}(r \mid \tilde{x}=g)=1-q,
$$

The existence of $w$ and $r$ are conditionally independent given $\tilde{x}$. If $r$ exists, $S$ is the first to hear it and can stop $r$ from reaching $R$.

The sequence of events is as follows. First, nature $(N)$ determines $\tilde{x}$. Second, $S$ is either attentive or not, which is unknown to $R$. An inattentive $S$ is out of the game from this point onward, so that $R$ will never get an official warning $w$ but will hear a rumor $r$ if it exists. An attentive $S$ issues $w$ to $R$ whenever she detects it, and decides whether to stop $r$ from reaching $R$ if it exists. Finally, $R$ decides whether to accept or reject $\tilde{x}$, and his payoff is realized.

The state space $\Omega$ includes all possible resolutions of the four uncertainties: the value of $\tilde{x}, S$ 's attentiveness, and the existence of $w$ and $r$. To denote a particular state, we stack together the resolutions of the four uncertainties in above order. For example, gaw means $\tilde{x}$ is good, $S$ is attentive, $w$ exists but $r$ does not. bir means $\tilde{x}$ is bad, $S$ is inattentive so there is no $w$, but $r$ exists. Therefore the state space is

$$
\Omega:=\{\text { gawr, gaw, gar, ga, gir, gi, bawr, baw, bar, ba, bir, bi }\} .
$$

Before $\tilde{x}$ is realized, $S$ and $R$ cannot differentiate between each pair of states that differ only by the value of $\tilde{x}$. It helps to group each such pair into an event:

$$
\begin{aligned}
a w r:= & \{g a w r, b a w r\}, a w:=\{g a w, b a w\}, \text { ar }:=\{g a r, b a r\}, \\
& a:=\{g a, b a\}, \text { ir }:=\{g i r, b i r\}, i:=\{g i, b i\} .
\end{aligned}
$$


One and only one of these six events will occur, which makes

$$
\Lambda:=\{a w r, a w, a r, a, i r, i\}
$$

a useful partition of $\Omega$ in our subsequent analysis.

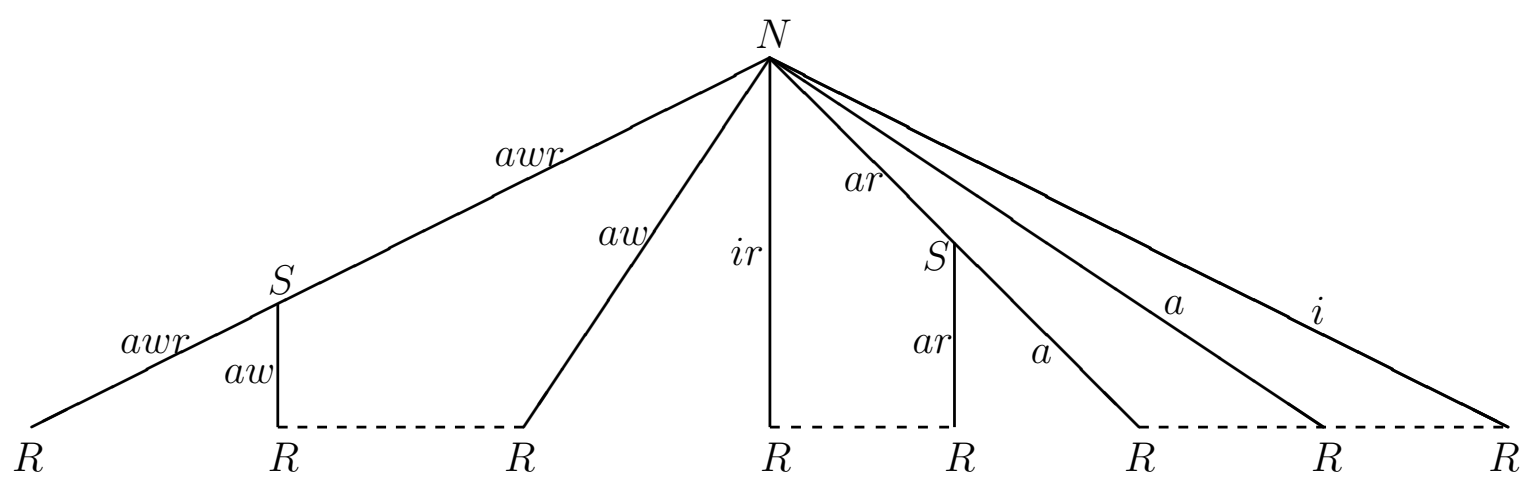

Figure 1: A Simplified Game Tree

Figure 1 shows a simplified game tree. $N$ decides how the four uncertainties resolve. $S$ can choose to squash $r$ when she is attentive in two events $a w r$ and $a r . R$ cannot tell whether $S$ is attentive unless $w$ exists. If $R$ hears no $r$, he cannot tell whether $r$ has been suppressed or it never existed. $R$ 's information sets are shown as the broken segments at the bottom of the game tree. Finally, $R$ makes his decision on $\tilde{x}$ and his payoff is realized.

$R$ 's expected payoff from $\tilde{x}$ given any information set $\mathcal{I}$ is

$$
[1-\operatorname{Pr}(b \mid \mathcal{I})] \times 1-\operatorname{Pr}(b \mid \mathcal{I}) \times c=1-(1+c) \operatorname{Pr}(b \mid \mathcal{I})
$$

which is positive if

$$
\operatorname{Pr}(b \mid \mathcal{I})<q^{*}:=\frac{1}{1+c}
$$

Therefore $R$ should choose $\tilde{x}$ if his belief on a bad $\tilde{x}$ is below $q^{*}$; otherwise he should avoids $\tilde{x}$.

Since the value of $\tilde{x}$ remains unknown till the end, a typical information set $\mathcal{I}$ that $R$ uses to make his decision is a subset of $\Lambda$. The conditioning information is more refined for a smaller set $\mathcal{I}$. $\Lambda$ itself represents the information set on which the prior belief is based:

$$
\operatorname{Pr}(b \mid \Lambda)=\operatorname{Pr}(b)=\frac{1}{2} .
$$

If $q^{*}<\frac{1}{2}$, then $R$ rejects $\tilde{x}$ given the prior and never reverts his decision because there can only be bad news $w$ and $r$ down the road. We thus focus on the more interesting case of $q^{*}>\frac{1}{2}$ so that $R$ would accept $\tilde{x}$ given his prior belief but may change his decision should bad news arrive. Suppose $q>q^{*}$ so that bad news $w$ or $r$ is material 
enough to alter $R$ 's decision. In sum, the maintained assumption is

$$
q^{*} \in\left(\frac{1}{2}, q\right)
$$

or equivalently $c<1<(1+c) q$.

We now find $R$ 's first-best decision that maximizes his welfare given each of the six events in $\Lambda$. Table 1 lists each event $E$, its probability $\operatorname{Pr}(E)$, the probability $\operatorname{Pr}(b \mid E)$ that $\tilde{x}$ is bad given $E$, whether $\operatorname{Pr}(b \mid E)>q^{*}$, and the sign of $\tilde{x}^{\prime}$ 's impact on $R$ 's welfare. The derivation of these results are in the Appendix. $R$ 's welfare from $\tilde{x}$ is negative in events awr and $i r$ but positive in every other event.

Table 1: $R$ 's Beliefs, First-Best Decisions, Welfare in Each Event $E \in \Lambda$

\begin{tabular}{|c|c|c|c|c|}
\hline \hline$E$ & $\operatorname{Pr}(E)$ & $\operatorname{Pr}(b \mid E)$ & vs $q^{*}$ & impact \\
\hline$a w r$ & $\frac{1}{2}\left[q^{2}+(1-q)^{2}\right] p$ & $\frac{q^{2}}{q^{2}+(1-q)^{2}}$ & $>q^{*}$ & - \\
\hline$a w$ & $(1-q) q p$ & $\frac{1}{2}$ & $<q^{*}$ & + \\
\hline$i r$ & $\frac{1}{2}(1-p)$ & $q$ & $>q^{*}$ & - \\
\hline$a r$ & $(1-q) q p$ & $\frac{1}{2}$ & $<q^{*}$ & + \\
\hline$i$ & $\frac{1}{2}(1-p)$ & $1-q$ & $<q^{*}$ & + \\
\hline$a$ & $\frac{1}{2}\left[q^{2}+(1-q)^{2}\right] p$ & $\frac{(1-q)^{2}}{q^{2}+(1-q)^{2}}$ & $<q^{*}$ & + \\
\hline \hline
\end{tabular}

Proposition 1. R's first-best is to avoid $\tilde{x}$ in events awr and ir and choose $\tilde{x}$ otherwise.

However, $R$ cannot tell ir from ar because in both events he only hears the rumor $r$ but not the warning $w$, and is therefore not sure if $S$ is attentive. This uncertainty leads to an inefficiency because the optimal decisions for $i r$ and ar differ.

\section{Inefficient Democratic Transparency}

When $S$ is attentive, she knows the existence of $w$ and $r$, and can therefore differentiate each event. Suppose $S$ is transparent and never interferes with the rumor. Then $R$ hears $r$ whenever it exists.

Lemma 1. If $E \in\{a w r, a w$, ar, $a\}$, then $S$ 's information set $\mathcal{I}_{S}=E$ and $\operatorname{Pr}\left(b \mid \mathcal{I}_{S}\right)=$ $\operatorname{Pr}(b \mid E)$. If $S$ never stops $r$, then

(a) If $E \in\left\{\right.$ awr, aw\}, then $R$ 's information set $\mathcal{I}_{R}=E$ and $\operatorname{Pr}\left(b \mid \mathcal{I}_{R}\right)=\operatorname{Pr}(b \mid E)$.

(b) If $R$ hears $r$ but not $w$, then $\mathcal{I}_{R}=\{$ ir, ar $\}$ and

$$
\operatorname{Pr}(b \mid\{i r, a r\})=\frac{(1-p q) q}{1-p+2(1-q) p q} \in\left(\frac{1}{2}, q\right)
$$

is decreasing in $p$ and increasing in $q$. 
(c) If $R$ hears nothing, then $\mathcal{I}_{R}=\{i, a\}$ and

$$
\operatorname{Pr}(b \mid\{i, a\})=\frac{(1-p q)(1-q)}{1-2(1-q) p q} \in\left(\frac{(1-q)^{2}}{q^{2}+(1-q)^{2}}, 1-q\right)
$$

is decreasing in $p$ and $q$.

Therefore $R$ 's information structure under transparency $\{a w r, a w,\{i r, a r\},\{i, a\}\}$ is cruder than $\Lambda$ since $R$ does not know if $S$ is attentive when he does not see $w$.

The comparative statics makes intuitive sense. $R$ 's belief on a bad $\tilde{x}$ given no warning is lower if $S$ is more likely attentive since the absence of $w$ more likely implies that an attentive $S$ detects no warning. $R$ 's belief given $r$ but not $w$ rises with $q$ the quality of information, because $S$ may be inattentive and not issue $w$ but the rumor shows up if it exists, so that $R$ should weigh $r$ more. $R$ 's belief given no news is lower if information is of higher quality as the lack of bad news becomes better news.

Table 2: Information Sets, Beliefs, Decisions, Welfare Under Transparency

\begin{tabular}{|c|c|c|c|c|c|c|c|c|}
\hline \hline$E$ & $\mathcal{I}_{S}$ & to $R$ & $\mathcal{I}_{R}$ & $\operatorname{Pr}(b \mid E)$ & $\operatorname{Pr}\left(b \mid \mathcal{I}_{S}\right)$ & $\operatorname{Pr}\left(b \mid \mathcal{I}_{R}\right)$ & vs $q^{*}$ & impact \\
\hline awr & $a w r$ & $w r$ & $a w r$ & $\frac{q^{2}}{q^{2}+(1-q)^{2}}$ & $\frac{q^{2}}{q^{2}+(1-q)^{2}}$ & $\frac{q^{2}}{q^{2}+(1-q)^{2}}$ & $>q^{*}$ & - \\
\hline$a w$ & $a w$ & $w$ & $a w$ & $\frac{1}{2}$ & $\frac{1}{2}$ & $\frac{1}{2}$ & $<q^{*}$ & + \\
\hline$i r$ & $\mathrm{n} / \mathrm{a}$ & $r$ & $\{i r, a r\}$ & $q$ & $\mathrm{n} / \mathrm{a}$ & $\in\left(\frac{1}{2}, q\right)$ & $?$ & $?$ \\
$a r$ & $a r$ & $r$ & $\{i r, a r\}$ & $\frac{1}{2}$ & $\frac{1}{2}$ & $\in\left(\frac{1}{2}, q\right)$ & $?$ & $?$ \\
\hline$i$ & $\mathrm{n} / \mathrm{a}$ & & $\{i, a\}$ & $1-q$ & $\mathrm{n} / \mathrm{a}$ & $<1-q$ & $<q^{*}$ & + \\
$a$ & $a$ & & $\{i, a\}$ & $\frac{(1-q)^{2}}{q^{2}+(1-q)^{2}}$ & $\frac{(1-q)^{2}}{q^{2}+(1-q)^{2}}$ & $<1-q$ & $<q^{*}$ & + \\
\hline \hline
\end{tabular}

Table 2 shows $S$ 's and $R$ 's information sets and beliefs as well as $R$ 's equilibrium decisions and welfare under democratic transparency where the rumor reaches $R$ freely. The column with header "to $R$ " describes what $R$ sees. $R$ 's non-singleton information sets $\{i r, a r\}$ and $\{i, a\}$ are in red and green respectively. $R$ cannot tell $i$ from $a$ but still makes the first-best decision of accepting $\tilde{x}$ because it is optimal in both events. It is because $R$ cannot distinguish $i r$ and $a r$ where different decisions are optimal that an inefficiency arises.

Theorem 2. There is always an inefficiency if $S$ never stops $r$.

(a) If $\operatorname{Pr}(b \mid\{$ ir, ar $\})<q^{*}$, then $R$ optimally accepts $\tilde{x}$ when he hears $r$ but not $w$. However, he is better off avoiding $\tilde{x}$ in event ir. His welfare loss relative to the first-best

$$
\frac{(1-p)[(c+1) q-1]}{2}>0
$$

is increasing in $q$ and decreasing in $p$.

(b) If $\operatorname{Pr}(b \mid\{$ ir, ar $\})>q^{*}$ then $R$ optimally avoids $\tilde{x}$ when he hears $r$ but not $w$. However, he is better off accepting $\tilde{x}$ in event ar. His welfare loss relative to the 
first-best

$$
\frac{(1-c)(1-q) p q}{2}>0
$$

is decreasing in $q \in\left(\frac{1}{2}, 1\right)$ and increasing in $p$.

It is no surprise that the comparative statics of the two cases are opposite. R's decision is right in one event but wrong in the other. Therefore a factor that reduces the welfare loss in one event increases the welfare loss in the other. The higher the information quality, the worse $R$ fares deciding against the rumor; the less likely that $S$ is attentive, the more likely that $R$ is facing event $i r$, which also aggravates $R$ 's expected loss in (12). The lower the information quality, the more likely that $S$ disagrees with $r$, making event ar more likely and $R$ 's expected loss in $(13)$ higher. A more likely attentive $S$ also raises the chance of ar relative to $i r$.

\section{Efficient Autocratic Opacity}

$S$ can avert $R$ 's welfare loss by choosing the right occasion to preemptively eliminate the rumor. $S$ has two decision points. First, in event awr where $S$ issues the official warning $w$ and hears the rumor $r, S$ 's information agrees with the rumor. Second, in event $a r$ where $S$ hears $r$ but has no $w, S$ disagrees with the rumor. Recall from Section 3 that if $S$ never stops the rumor, then $R$ cannot tell $i r$ and ar apart. Since $R$ 's first-best decisions are different in events $i r$ and $a r, R$ makes the wrong decision in one event due to his inference problem.

Consider $S$ 's autocratic opacity to only quell the rumor when she disagrees with it. Then $r$ reaches $R$ in event awr but not ar. Now $R$ knows that if he hears $r$ but not $w$, then ir has occurred. This separates $R$ 's information set $\{i r, a r\}$ and prevents the information loss that leads to $R$ 's inference problem and inefficient decision in Section 3. Therefore $S$, by stopping the dissident rumor, helps $R$ tell event $i r$ from $a r$.

Table 3: $R$ 's Information, Beliefs, Decisions, Welfare Under Autocratic Opacity

\begin{tabular}{|c|c|c|c|c|c|c|c|}
\hline \hline$E$ & $\mathcal{I}_{S}$ & to $R$ & $\mathcal{I}_{R}$ & $\operatorname{Pr}(b \mid E)$ & $\operatorname{Pr}\left(b \mid \mathcal{I}_{R}\right)$ & vs $q^{*}$ & impact \\
\hline$a w r$ & $a w r$ & $w r$ & $a w r$ & $\frac{q^{2}}{q^{2}+(1-q)^{2}}$ & $\frac{q^{2}}{q^{2}+(1-q)^{2}}$ & $>q^{*}$ & - \\
$a w$ & $a w$ & $w$ & $a w$ & $\frac{1}{2}$ & $\frac{1}{2}$ & $<q^{*}$ & + \\
$i r$ & $\mathrm{n} / \mathrm{a}$ & $r$ & $i r$ & $q$ & $q$ & $>q^{*}$ & - \\
\hline$a r$ & $a r$ & & $\{a r, i, a\}$ & $\frac{1}{2}$ & $1-q$ & $<q^{*}$ & + \\
$i$ & $\mathrm{n} / \mathrm{a}$ & & $\{a r, i, a\}$ & $1-q$ & $1-q$ & $<q^{*}$ & + \\
$a$ & $a$ & & $\{a r, i, a\}$ & $\frac{(1-q)^{2}}{q^{2}+(1-q)^{2}}$ & $1-q$ & $<q^{*}$ & + \\
\hline \hline
\end{tabular}

There is no free lunch to eliminate uncertainty, however. $S$ 's strategy brings in a new inference problem. When $R$ receives nothing, he cannot tell whether the rumor has been suppressed (event $a r$ ) or it never existed (events $i$ and $a$ ); neither does $R$ know whether $S$ is attentive. Therefore $a r$ joins $\{i, a\}$ to form $R$ 's new non-singleton 
information set $\{a r, i, a\}$. Fortunately, the three events inside share the same first-best decision. Therefore $R$ 's new inference problem does not cause any inefficiency.

Theorem 3. Suppose $S$ quiets $r$ only in event ar.

(a) If $E \in\{$ ir, awr, aw $\}$, then $\mathcal{I}_{R}=E$ and $\operatorname{Pr}\left(b \mid \mathcal{I}_{R}\right)=\operatorname{Pr}(b \mid E)$.

(b) If $R$ receives nothing, then $\mathcal{I}_{R}=\{a r, i, a\}$ and $\operatorname{Pr}\left(b \mid \mathcal{I}_{R}\right)=1-q<q^{*}$.

$S$ helps $R$ attain the first-best by her autocratic opacity of stopping dissident rumor.

$R$ 's information structure $\{a w r, a w, i r,\{a r, i, a\}\}$ when $S$ quiets dissident rumor is cruder than $\Lambda$. Table 3 summarizes $R$ 's information, beliefs, equilibrium decisions, and welfare. $R$ 's non-singleton information set $\{a r, i, a\}$ is in green as $R$ makes the firstbest decision. Figure 2 shows $S$ 's equilibrium decisions in red. $S$ effectively practices autocratic opacity to conveyed welfare-relevant information to $R$ while giving up transmitting welfare-irrelevant information. Benevolent authorities can restore efficiency by collecting first-hand information via surveillance and stopping dissident rumor from reaching the receiver.

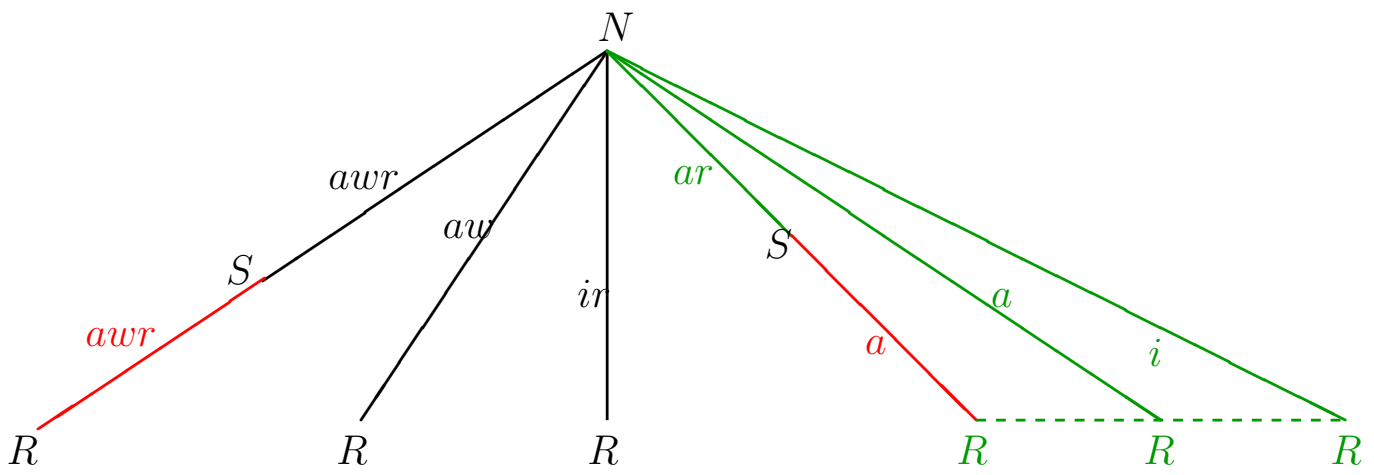

Figure 2: The Equilibrium and $R$ 's Information Sets Under Autocratic Opacity

\section{Predictions}

Our theory has policy implications in law and political economy. It is permissible for benevolent authorities to run surveillance programs such as PRISM leaked by Edward Snowden to be the first to know. It can be optimal for them to exercise autocratic opacity by muzzling dissident voices and controlling information flow to the public for purpose of attaining efficiency.

Implication. It is optimal for authorities to monitor and suppress dissident rumor.

In practice, however, some authorities are less able to suppress dissident information due to disclosure laws, human rights, or implementation difficulties. For example, democratic and transparent nations have less leeway than autocratic and opaque regimes to muzzle dissident opinions. Moreover, it is harder for larger countries with bigger populations, decentralized organizations, and better communication channels to withhold information. Therefore we have the unconventional prediction below. 
Prediction 1. Members of small, autocratic, opaque, centralized entities with weaker disclosure laws benefit more from autocratic opacity than members of large, democratic, transparent, decentralized organizations.

Comparative statics in Lemma 1 and Theorem 2 yields predictions on how $R$ likely errs if $S$ is unable to withhold information to improve $R$ 's decision. If $R$ makes a wrong decision, the direction of his error and his welfare loss are eventually realized. A wrong decision is either aggressively accepting $\tilde{x}$ in event ir or conservatively avoiding $\tilde{x}$ in event $a r$ by Theorem 2. Given that $R$ optimally chooses $\tilde{x}$ when his belief $\operatorname{Pr}(b \mid\{i r, a r\})$ is low and this belief is decreasing in $p$ and increasing in $q$ by Lemma 1, $R$ more likely errs aggressively if $S$ is more likely attentive or if information has lower quality. Theorem 2 gives similar comparative statics on welfare loss. Section 3 offers intuitions behind all comparative statics.

Prediction 2. Suppose $R$ makes a wrong decision. The error is more likely aggressive acceptance and the associated welfare loss is higher for a more attentive $S$ or noisier information. The error is more likely conservative avoidance and the associated welfare loss is lower for a busier $S$ or more accurate information.

To connect Prediction 2 to practice, busier nations at war or with larger populations are less able to stay attentive to $R$ 's decisions. More transparent nations with good research institutions are better able to generate high quality information. Information quality also improves over time with better technology.

\section{Robustness}

Binary news is more general than it seems, as a continuous $\tilde{x}$ can be discretized and in practice only values beyond a certain threshold make bad news. The model can be rewritten to have good and bad news with the same moral. The assumption that $R$ does not know if $S$ is attentive is nonessential for our result and below we present a simple model without this structure.

There are two independent random signals: $\tilde{\alpha}, \tilde{\beta} \in\{0,1\}$. R's payoff is 0 if he knows both signals correctly but -1 otherwise. $S$ and $R$ observe $\tilde{\alpha}$ and $\tilde{\beta}$ but $R$ misinterprets $\tilde{\beta}$ as $1-\tilde{\beta}$ with probability $\rho<\frac{1}{2}$ independent of $\tilde{\alpha}$ and $\tilde{\beta}$. The first-best is for $R$ to know $\tilde{\alpha}$ and $\tilde{\beta}$ to attain 0 welfare.

If $S$ does nothing, then $R$ misinterprets $\tilde{\beta}$ with probability $\rho$ for an expected payoff of $-\rho<0$. By wisely suppressing $\tilde{\beta}$ contingently, $S$ can restore efficiency. Suppose $S$ withholds $\tilde{\beta}$ if and only if $\tilde{\beta} \neq \tilde{\alpha}$. Given $S$ 's equilibrium strategy, $R$ know that $\tilde{\beta}=\tilde{\alpha}$ whenever he sees $\tilde{\beta}$ and $\tilde{\beta} \neq \tilde{\alpha}$ whenever he does not see $\tilde{\gamma}$. $R$ reads $\tilde{\alpha}$ correctly and infers $\tilde{\beta}$.

Proposition 2. $S$ restores the first-best by stopping $\tilde{\beta}$ from reaching $R$ if $\tilde{\beta} \neq \tilde{\alpha}$.

Intuitively, it makes sense for $S$ to contingently withhold $\tilde{\beta}$ that $R$ may misinterpret, so that he infers it from $\tilde{\alpha}$ which he reads correctly. 


\section{Conclusions}

A benevolent but possibly inattentive sender can exercise autocratic opacity to monitor and squash dissident yet accurate rumor so as to swap out the more welfare-relevant uncertainty to help the receiver make an efficient decision. We show the inefficiency of transparency and rationalize surveillance programs, dissident rumor suppression, and opaqueness by authorities. Our analysis has applications, predictions, and policy implications in law and governance. The paper offers a distinct situation where the sender's incentive is aligned with the receiver's, more information helps the receiver, yet it is optimal for the sender to stop some information from reaching the receiver in order to attain efficiency. 


\section{Appendix: Proofs}

Derivation of Table 1. $\tilde{x}$ is good with probability $\frac{1}{2}$; given this, $r$ exists with probability $1-q$; independently with probability $p, S$ is attentive; given this and the good $\tilde{x}, w$ exists with probability $1-q$. Hence

$$
\operatorname{Pr}(\text { gawr })=\frac{1}{2}(1-q)^{2} p .
$$

Similarly, $\operatorname{Pr}($ bawr $)=\frac{1}{2} q^{2} p$. Therefore

$$
\operatorname{Pr}(a w r)=\operatorname{Pr}(b a w r)+\operatorname{Pr}(\text { gawr })=\frac{1}{2}\left[q^{2}+(1-q)^{2}\right] p .
$$

Likewise,

$$
\begin{aligned}
\operatorname{Pr}(a w) & =\operatorname{Pr}(\text { gaw })+\operatorname{Pr}(\text { baw })=\frac{1}{2}(1-q) p q+\frac{1}{2}(1-q) p q=(1-q) p q \\
\operatorname{Pr}(\text { ir }) & =\operatorname{Pr}(\text { gir })+\operatorname{Pr}(\text { bir })=\frac{1}{2}(1-q)(1-p)+\frac{1}{2}(1-p) q=\frac{1-p}{2} \\
\operatorname{Pr}(a r) & =\operatorname{Pr}(\text { gar })+\operatorname{Pr}(\text { bar })=\frac{1}{2}(1-q) p q+\frac{1}{2}(1-q) p q=(1-q) p q \\
\operatorname{Pr}(i) & =\operatorname{Pr}(\text { gi })+\operatorname{Pr}(\text { bi })=\frac{1}{2}(1-p) q+\frac{1}{2}(1-q)(1-p)=\frac{1-p}{2} \\
\operatorname{Pr}(a) & =\operatorname{Pr}(\text { ga })+\operatorname{Pr}(b a)=\frac{1}{2} q^{2} p+\frac{1}{2}(1-q)^{2} p=\frac{1}{2}\left[q^{2}+(1-q)^{2}\right] p .
\end{aligned}
$$

By the law of conditional probability,

$$
\operatorname{Pr}(b \mid a w r)=\frac{\operatorname{Pr}(b a w r)}{\operatorname{Pr}(a w r)}=\frac{q^{2}}{q^{2}+(1-q)^{2}}>q>q^{*} .
$$

To see the inequality, note since $q \in\left(\frac{1}{2}, 1\right)$, we have $(q-1)(2 q-1)<0$, or $q^{2}+(1-q)^{2}<$ $q$, or $\operatorname{Pr}(b \mid a w r)>q$. Likewise,

$$
\operatorname{Pr}(b \mid a)=\frac{\operatorname{Pr}(b a)}{\operatorname{Pr}(a)}=\frac{(1-q)^{2}}{q^{2}+(1-q)^{2}}<\frac{(1-q)^{2}}{(1-q)^{2}+(1-q)^{2}}=\frac{1}{2}<q^{*} .
$$

Similarly,

$$
\operatorname{Pr}(b \mid a w)=\frac{\operatorname{Pr}(b a w)}{\operatorname{Pr}(a w)}=\frac{1}{2}<q^{*} .
$$

The same is true of $\operatorname{Pr}(b \mid a r)$. We also have

$$
\operatorname{Pr}(b \mid i r)=\frac{\operatorname{Pr}(b i r)}{\operatorname{Pr}(i r)}=q>q^{*}, \quad \operatorname{Pr}(b \mid i)=\frac{\operatorname{Pr}(b i)}{\operatorname{Pr}(i)}=1-q<\frac{1}{2}<q^{*} .
$$

Finally, the sign of $R$ 's welfare follows by (7).

Lemma 1 Proof. If event $i r$ or $i$ occurs then $S$ is inattentive and has no belief. 
Among the six events in $\Lambda$, the unique event where $S$ sees both $w$ and $r$ is awr; the unique event where it sees $w$ but not $r$ is aw; the unique event where it has no $w$ but hears $r$ is ar; the unique event where it has no news is $a$. Therefore $S$ can distinguish each of the four events awr, aw, ar, a. So if $E \in\{a w r, a w, a r, a\}, \mathcal{I}_{S}=E$ and $S$ 's belief updates to $\operatorname{Pr}\left(b \mid \mathcal{I}_{S}\right)=\operatorname{Pr}(b \mid E)$. We next compute $R$ 's beliefs.

(a). Among the six events in $\Lambda$, the unique event where $R$ sees $w$ and $r$ is awr; the unique event where $R$ sees $w$ but not $r$ is aw. Therefore $R$ can distinguish awr and $a w$. So if $E \in\{a w r, a w\}, \mathcal{I}_{R}=E$ and $R$ 's belief updates to $\operatorname{Pr}\left(b \mid \mathcal{I}_{R}\right)=\operatorname{Pr}(b \mid E)$.

(b). There are two events $i r$ and ar among the six in $\Lambda$ where $R$ hears $r$ but not $w$. $R$ cannot tell $i r$ from ar because he does not know if $S$ is attentive. So $\mathcal{I}_{R}=\{i r, a r\}$ if either occurs. $R$ 's belief updates to

$$
\operatorname{Pr}(b \mid\{i r, a r\})=\frac{\operatorname{Pr}(b i r)+\operatorname{Pr}(b a r)}{\operatorname{Pr}(i r)+\operatorname{Pr}(a r)}=\frac{(1-p q) q}{1-p+2(1-q) p q} \in\left(\frac{1}{2}, q\right) .
$$

$\operatorname{Pr}(b \mid\{i r, a r\})>\frac{1}{2}$ reduces to $2 q>1-p+2 p q$, or $(1-p)(2 q-1)>0$, which holds by assumption. $\operatorname{Pr}(b \mid\{i r, a r\})<q$ reduces to $(1-q) p<2(1-q) p q$, or $1<2 q$. $\operatorname{Pr}(b \mid\{i r, a r\})$ is decreasing in $p$ because

$$
\frac{\partial}{\partial p}\left[\frac{(1-p q) q}{1-p+2(1-q) p q}\right]=-\frac{(2 q-1)(1-q) q}{[1-p+2(1-q) p q]^{2}}<0 .
$$

$\operatorname{Pr}(b \mid\{i r, a r\})$ is increasing in $q$ because

$$
\frac{\partial}{\partial q}\left[\frac{(1-p q) q}{1-p+2(1-q) p q}\right]=\frac{[1-2(1-q) p q](1-p)}{[1-p+2(1-q) p q]^{2}}>0 .
$$

The numerator is positive if $2(1-q) p q<1$, which holds since $2(1-q)<1$.

(c). There are two events $i$ and $a$ among the six in $\Lambda$ where $R$ hears nothing. $R$ cannot tell $i$ from $a$ because he does not know if $S$ is attentive. So $\mathcal{I}_{R}=\{i, a\}$ if either occurs. $R$ 's belief updates to

$$
\operatorname{Pr}(b \mid\{i, a\})=\frac{\operatorname{Pr}(b i)+\operatorname{Pr}(b a)}{\operatorname{Pr}(i)+\operatorname{Pr}(a)}=\frac{(1-p q)(1-q)}{1-2(1-q) p q} \in\left(\frac{(1-q)^{2}}{(1-q)^{2}+q^{2}}, 1-q\right) .
$$

Given $2(1-q) p q<1$, we cross-multiply and reduce the lower-bound inequality to $(1-p q)\left[(1-q)^{2}+q^{2}\right]>(1-q)[1-2(1-q) p q]$, or $2(1-p) q^{2}>(1-p) q$, or $2 q>1$ which holds by assumption. The upper-bound inequality $\operatorname{Pr}(b \mid\{i, a\})<1-q$ reduces to $1-p q<1-2(1-q) p q$, or $p q>2(1-q) p q$, or $2 q>1$. $\operatorname{Pr}(b \mid\{i, a\})$ is decreasing in $p$ because

$$
\frac{\partial}{\partial p}\left[\frac{(1-p q)(1-q)}{1-2(1-q) p q}\right]=-\frac{(2 q-1)(1-q) p}{[1-2(1-q) p q]^{2}}<0
$$


$\operatorname{Pr}(b \mid\{i, a\})$ is decreasing in $q$ because

$$
\frac{\partial}{\partial q}\left[\frac{(1-p q)(1-q)}{1-2(1-q) p q}\right]=-\frac{1-p+2 p q(1-q)}{\left(1-2 p q+2 q^{2} p\right)^{2}}<0 .
$$

Theorem 2 Proof. If $\operatorname{Pr}(b \mid\{i r, a r\})<q^{*}$, then $R$ optimally chooses $\tilde{x}$ when he hears $r$ but not $w . R$ makes the wrong decision in event $i r$ since the first-best there is to avoid $\tilde{x}$. His welfare from $\tilde{x}$ in event $i r$ is $[1-\operatorname{Pr}(b \mid i r)] \times 1-\operatorname{Pr}(b \mid i r) \times c=1-(1+c) q<0$. Since $\operatorname{Pr}(i r)=\frac{1-p}{2}, R$ 's expected welfare loss relative to the first-best is

$$
\frac{(1-p)[(c+1) q-1]}{2}>0
$$

which is increasing in $q$ and decreasing in $p$.

If $\operatorname{Pr}(b \mid\{i r, a r\})>q^{*}$, then $R$ optimally avoid $\tilde{x}$ when he hears $r$ but not $w . R$ makes the wrong decision in event ar since the first-best there is to choose $\tilde{x}$. His welfare from $\tilde{x}$ in event $a r$ is $[1-\operatorname{Pr}(b \mid a r)] \times 1-\operatorname{Pr}(b \mid a r) \times c=\frac{1-c}{2}>0$. Since $\operatorname{Pr}(a r)$ $=(1-q) p q, R$ 's expected welfare loss relative to the first-best is

$$
\frac{(1-c)(1-q) p q}{2}>0,
$$

which is decreasing in $q \in\left(\frac{1}{2}, 1\right)$ and increasing in $p$.

Therefore an inefficiency always exists.

Theorem 3 Proof. The only event where $R$ hears both $w$ and $r$ is awr; the only event where $R$ hears $w$ but not $r$ is aw; the only event where $R$ hears $r$ but not $w$ is $i r$. So $R$ can distinguish each of $a w r, a w$, and $i r$. If $E \in\{a w r, a w, i r\}$, then $R$ 's information set $\mathcal{I}_{R}=E$, he updates belief to $\operatorname{Pr}\left(b \mid \mathcal{I}_{R}\right)=\operatorname{Pr}(b \mid E)$, and makes the first-best decision.

$R$ cannot distinguish $a r, i, a$ because he receives nothing in these events. So if $E \in\{a r, i, a\}$, then $R$ 's information set is $\mathcal{I}_{R}=\{a r, i, a\}$ and his belief updates to

$$
\operatorname{Pr}(b \mid\{a r, i, a\})=\frac{\operatorname{Pr}(b a r)+\operatorname{Pr}(b i)+\operatorname{Pr}(b a)}{\operatorname{Pr}(i r)+\operatorname{Pr}(i)+\operatorname{Pr}(a)}=1-q<\frac{1}{2}<q^{*} .
$$

$R$ optimally accept $\tilde{x}$, which is the first-best in each of the three events $a r, i$, and $a$.

Therefore the first-best is attained in every event if $S$ stops dissident rumor. 


\section{References}

[1] Amador, Manuel, and Pierre-Olivier Weill. 2012. Learning from Private and Public Observations of Others' Actions. Journal of Economic Theory 147(3):910-940.

[2] Angeletos, George-Marios, and Alessandro Pavan. 2007. Efficient Use of Information and Social Value of Information. Econometrica 75(4):1103-1142.

[3] Burguet, Roberto, and Xavier Vives. 2000. Social Learning and Costly Information Acquisition. Economic Theory 15(1):185-205.

[4] Crawford, Vincent P., and Joel Sobel. 1982. Strategic Information Transmission. Econometrica 50(6):1431-1451.

[5] Dewatripont, Mathias, and Jean Tirole. 2005. Modes of Communication. Journal of Political Economy 113(6):1217-1238.

[6] Dye, Ronald A. 1985a. Disclosure of Nonproprietary Information. Journal of Accounting Research 23(1):123-145.

[7] Dye, Ronald A. 1985b. Strategic Accounting Choice and the Effects of Alternative Financial Reporting Requirements. Journal of Accounting Research 23(2):544-574.

[8] Dye, Ronald A. 1986. Proprietary and Nonproprietary Disclosures. Journal of Business 59(2):331-336.

[9] Gonedes, Nicholas J. 1980. Public Disclosure Rules, Private InformationProduction Decisions, and Capital Market Equilibrium. Journal of Accounting Research 18(2):441-476.

[10] Gonedes, Nicholas J., and Nicholas Dopuch. 1974. Capital Market Equilibrium, Information Production, and Selecting Accounting Techniques: Theoretical Framework and Review of Empirical Work. Journal of Accounting Research 12(3):48-129

[11] Grossman, Sanford J. 1981. The Informational Role of Warranties and Pivate Disclosures about Poduct Quality. Journal of Law and Economics 24(3):461-483.

[12] Grossman, Sanford J., and Oliver D. Hart. 1980. Disclosure Laws and Takeover Bids. Journal of Finance 35(2):323-334.

[13] Hirshleifer, Jack. 1971. The Pivate and Social Value of Information and the Reward to Inventive Activity. American Economic Review 61(4):561-574.

[14] Milgrom, Paul R. 1981. Good News and Bad News: Representation Theorems and Applications. Bell Journal of Economics 12(2):380-391.

[15] Morris, Stephen, and Hyun Song Shin. 2002. Social Value of Public Information. American Economic Review 92(5):1521-1534.

[16] Teoh, Siew Hong. 1997. Information Disclosure and Voluntary Contributions to Public Goods. RAND Journal of Economics 28(3):385-406. 